\title{
Serum Levels of Vitamin D in Diabetic Patients With and Without Retinopathy
}

\author{
Mehrdad Afarid', MD; Naghme Ghattavi ${ }^{2}$, MD; Mohammadkarim Johari ${ }^{1}$, MD \\ ${ }^{1}$ Poostchi Ophthalmology Research Center, Department of Ophthalmology, School of Medicine, Shiraz University of Medical \\ Sciences, Shiraz, Iran \\ ${ }^{2}$ Student Research Committee, Shiraz University of Medical Sciences, Shiraz, Iran \\ ORCID: \\ Mehrdad Afarid: https://orcid.org/0000-0003-2348-9163 \\ Mohammadkarim Johari: https://orcid.org/0000-0002-5442-0073
}

\section{Abstract}

Purpose: To evaluate the levels of vitamin $D$ in the serum of diabetic patients with and without diabetic retinopathy (DR).

Methods: Thirty patients with DR and thirty diabetic patients without retinopathy were included in this cross-sectional study. Based on ophthalmic examination, patients with DR were categorized into having non-proliferative retinopathy (NPDR) and proliferative retinopathy (PDR). Patients were tested for fasting blood sugar (FBS), hemoglobin A1C (HbA1C), 25-hydroxy vitamin D (25 (OH) D), and creatinine levels in the serum, and for urine protein. Vitamin D deficiency was defined as a serum $25(\mathrm{OH})$ D level $<20 \mathrm{ng} / \mathrm{mL}$. Results: We found that all diabetic patients had mild vitamin D deficiency (serum $25(\mathrm{OH})$ D level $=10-20 \mathrm{ng} / \mathrm{mL}$ ). The mean serum $25(\mathrm{OH}) \mathrm{D}$ concentration in patients with DR was lower than in those without DR $(12.10 \pm 14.62 \mathrm{ng} / \mathrm{mL}$ vs $15.61 \pm 9.40 \mathrm{ng} / \mathrm{mL}$, respectively, $P=0.012$ ). Trace or more proteinuria was frequently present in patients with $\mathrm{DR}$ than in those without DR (56\% in DR vs $30 \%$ in non-DR; $P=0.037)$. There were no significant differences in the FBS, HbA1C, and serum creatinine levels between patients with or without retinopathy.

Conclusion: The present study demonstrated that patients with DR had lower levels of serum vitamin $D$ compared with those without retinopathy.

Keywords: Diabetes Mellitus; Diabetic Retinopathy; 25-Hydroxy Vitamin D

J Ophthalmic Vis Res 2020; 15 (2): 172-177

\section{INTRODUCTION}

The prevalence of diabetes mellitus, a metabolic syndrome with several complications, has

\section{Correspondence to:}

Mohammadkarim Johari, MD. Poostchi Ophthalmology Research Center, Department of Ophthalmology, School of Medicine, Shiraz University of Medical Sciences, Poostchi St., Shiraz 714737, Iran.

E-mail: mkjoharii@gmail.com

Received: 21-10-2018 Accepted: 23-10-2019

\section{Access this article online}

Website: https://knepublishing.com/index.php/JOVR

DOI: 10.18502/jovr.v15i2.6734 increased worldwide in the recent decades. ${ }^{[1]}$ Based on the latest global burden of disease estimates in 2011 (SuRFNCD-2011), the prevalence of type 2 diabetes in the Iranian population is $11 \% .{ }^{[2]}$

Diabetic retinopathy (DR) is a major complication of diabetes mellitus that can result in retinal vascular abnormalities and severe visual

This is an open access journal, and articles are distributed under the terms of the Creative Commons Attribution-NonCommercial-ShareAlike 4.0 License, which allows others to remix, tweak, and build upon the work non-commercially, as long as appropriate credit is given and the new creations are licensed under the identical terms. 
impairment. DR is defined as a vascular disease resulting from hyperglycemia, and is characterized by altered structure of retinal endothelial vessels and disrupted blood-retinal barrier. The retinal vasculature is gradually damaged resulting in ischemic changes that lead to the formation of new vessels and its consequences. ${ }^{[3]}$

Different studies have reported abnormalities in the metabolism of calcium, phosphate, and vitamin $D$ in diabetic patients. Bayani et al showed that vitamin $\mathrm{D}$ concentration was significantly lower in diabetic patients compared with that in healthy individuals. ${ }^{[4]}$ Moreover, animal and human studies have demonstrated a correlation between vitamin $D$ deficiency and impaired insulin production and secretion as well as insulin sensitivity. ${ }^{[5-7]}$ Another study reported that reduced serum insulin levels could be related to the lower level of $1,25(\mathrm{OH})_{2} \mathrm{D} .^{[8]}$

Vitamin $D$ has an inhibitory effect on vascular angiogenesis. ${ }^{[9]}$ It is believed that vitamin $D$ deficiency, which is described as a serum 25 $(\mathrm{OH})$ D concentration $<20 \mathrm{ng} / \mathrm{mL}$, affects the pathogenesis and progression of DR. ${ }^{[10]}$

Vitamin D deficiency is a common public health concern. A study reports that the prevalence of vitamin $D$ deficiency varies across different populations. ${ }^{[11]}$ The prevalence of vitamin $D$ deficiency in the United States was reported to be $41.6 \%$ in $2005 .^{[12]}$ Additionally, individuals who had no college education and had low serum levels of high-density lipoprotein cholesterol, high body mass index (BMI), hypertension or were on low calcium diet had low serum vitamin $D$ levels. ${ }^{[12]} A$ study in Tehran, Iran reported the prevalence of vitamin $D$ deficiency to be $43.3 \%$ among adolescents, with higher rates in females compared to males. ${ }^{[13]}$

The most likely crucial role of Vitamin $D$ related to diabetic pathogenicity and its main complications such as DR is its involvement in several body mechanisms such as adjustment of blood glucose or body vascular consistency. Therefore, we decided to investigate the serum levels of vitamin $D$ in our diabetic patients with and without DR in Fars Province, Iran.

\section{METHODS}

This cross-sectional study was conducted on patients with type 2 diabetes who were referred to the ophthalmology clinics of the Shiraz University of Medical Sciences. The study was approved by the Shiraz University of Medical Sciences Ethics Committee. Thirty patients with DR and thirty patients without retinopathy were included. Patients with a history of smoking, chronic kidney disease, active infection, liver disease, primary or secondary hyperparathyroidism, serum creatinine levels $>2 \mathrm{mg} / \mathrm{dL}$, and those receiving vitamin $D$, calcium supplements, or any other medication that could change vitamin D metabolism, such as rifampin or phenytoin, were excluded from the study. Patients who had a history of bone fracture or orthopedic surgeries during the past year were also excluded.

Age, sex, and duration of diabetes were recorded for all subjects. Patients were categorized into two groups based on the duration of diabetes $(<10$ years and $>10$ years).

All patients underwent complete ophthalmic examination by a vitreoretinal surgeon for diagnosing DR; this examination consisted of funduscopy with a slit-lamp and $90 \mathrm{D}$ lenses and an indirect ophthalmoscopy. DR was diagnosed in the presence of one or more of the following signs: microaneurysms, cotton-wool spots, intraretinal hemorrhages, or macular edema. DR was categorized into mild to severe non-proliferative diabetic retinopathy (NPDR) based on the absence of any neovascularization and proliferative diabetic retinopathy (PDR). Mild NPDR was defined as having only microaneurysms, whereas severe NPDR was defined as having any one of the following features: severe intraretinal hemorrhages in four quadrants, venous beading in two or more quadrants and intraretinal microvascular anomalies (IRMAs) in one or more quadrants; moderate NPDR was between the above two categories. In cases of asymmetrical retinopathy, the patient was assigned to the group corresponding to the eye with the more severe retinopathy scale.

Blood samples were drawn in the morning after a 12-h fast. Fasting blood sugar (FBS) and glycated hemoglobin $\mathrm{A} 1 \mathrm{C}(\mathrm{HbA1C})$ and serum calcium, phosphorus, and creatinine levels were measured. The presence of protein in the urine was checked with dipstick method.

Serum vitamin $D$ level was assessed measuring the level of serum 25-OH vitamin D. The 25 $(\mathrm{OH}) \mathrm{D}$ levels were measured from serum samples using solid phase enzyme-linked immunosorbent assay (DRG $25(\mathrm{OH})$ D total ELISA Kit; DRG Instruments $\mathrm{GMBH}$, Germany). The serum concentration 
$>30 \mathrm{ng} / \mathrm{mL}$ was considered as sufficient. Cutoffs for serum vitamin $D$ concentration status are described in Table 1.

\section{Statistical Analysis}

SPSS 17.0 software for Windows (SPSS Inc., Chicago, IL, USA) was used for data analysis. The variables with a normal distribution are shown as mean \pm standard deviation, and nominal variables are expressed as number and percentage. The normality assumption in continuous variables was evaluated using the Kolmogorov-Smirnov test. Distributions higher than $P>0.05$ were accepted as normally distributed variables. The differences between normally distributed and continuous independent variables were assessed by the independent samples $t$-test. Those without a normal distribution were compared using the Mann-Whitney $U$ test between the groups. Categorical variables were compared using the Chi-squared test. For all comparisons, $P<0.05$ was considered significant.

\section{RESULTS}

The study included 15 female subjects with mean age ( \pm standard deviation) of $60.13 \pm 6.02$ years and 15 males with mean age of $57.60 \pm 5.43$ years in the group with DR and 16 females with mean age of $59.05 \pm 6.25$ years and 14 males with mean age of $57.30 \pm 8.50$ years in the group without DR. There was no statistically significant difference between the groups regarding their age $(P=0.73)$ and $\operatorname{sex}(P=0.75)$.

The demographic and clinical characteristics of the patients in the study are shown in Table 2 .

Among patients with DR, 21 patients had NPDR (13 mild to moderate and 8 severe) and 9 patients had PDR. All patients had mild vitamin D deficiency. The mean serum vitamin $D$ concentration in patients with DR $(12.10 \pm 14.62 \mathrm{ng} / \mathrm{mL}$; NPDR and PDR) was lower than in those without DR (15.61 \pm $9.40 \mathrm{ng} / \mathrm{mL})(P=0.012)$. Regression analysis with independent variables (FBS, duration of diabetes and insulin use) showed similar results $(P=0.031)$.

The mean $25(\mathrm{OH}) \mathrm{D}$ concentration between the DR subgroups (severe NPDR and PDR vs mild to moderate NPDR) was $10.51 \pm 10.45 \mathrm{ng} / \mathrm{mL}$ and 13.45 $\pm 8.4 \mathrm{ng} / \mathrm{mL}$, respectively $(P=0.681)$.

Serum $25(\mathrm{OH})$ D concentration in all patients was compared among the age groups. The mean \pm
SD in patients with age ranging from 41 to 50 years, 51 to 60 years, and $\geq 60$ years were $10.11 \pm 9.50$, $14.15 \pm 16.06,14.99 \pm 6.94$, respectively $(P=0.128)$.

Regarding the treatment, $46 \%$ of patients without DR and $86.6 \%$ of patients with DR were taking insulin. Univariate analysis between the two groups showed that the use of insulin in patients with DR was significantly higher than in those without DR $(P$ $=0.014$ ).

The duration of diabetes was different between the groups. Nine (30\%) patients with no diabetic retinopathy (NDR) had diabetes for more than 10 years and 16 (53\%) patients with DR had diabetes for more than 10 years. However, the difference was not statistically significant $(P=0.081)$.

Binomial variable multiple logistic regression analysis with independent variables revealed that FBS, HbA1C, BUN, serum creatinine, and serum vitamin $D$ levels did not show the duration of diabetes as an independent risk factor for DR $(P=$ $0.067)$. However, this value was significant in terms of the type of treatment (insulin use or not) $(P=$ 0.005).

Patients with DR had a higher positive dipstick test rate for proteinuria than those without DR (56\% of the patients with DR had traces of or more proteinuria vs $30 \%$ in no DR; $P=0.037$ ).

FBS, serum BUN, and creatinine levels were not different between the patients with or without retinopathy. Although vitamin $D$ may influence the levels of serum calcium, our data showed no significant difference in serum calcium or phosphorus between the two groups $(P=0.511$ and $P=0.416$, respectively).

\section{DISCUSSION}

In the current study, we evaluated the serum levels of vitamin D in diabetic patients with and without DR. Furthermore, we investigated other possible factors that could influence the progression of DR. Similar to previous studies, all included patients had $25(\mathrm{OH}) \mathrm{D}$ deficiency. ${ }^{[15-17]}$ The current study showed that the mean serum $25(\mathrm{OH})$ D concentration in patients with DR was lower than in those without DR, especially those with severe NPDR and PDR. These results were comparable to other studies. For example, Payne et al found that diabetic patients, especially those with PDR, had lower 25 $(\mathrm{OH}) \mathrm{D}$ levels than those without diabetes. ${ }^{[19]} \mathrm{Sim}-$ ilarly, Luo et al in their meta-analysis that included 
Table 1. Serum 25(OH) D concentration ${ }^{[14}$

25(OH) D concentration

25(OH) D status

$\leq 10 \mathrm{ng} / \mathrm{mL}$

Severe deficiency

$10-20 \mathrm{ng} / \mathrm{mL}$

Mild deficiency

20-30 ng/mL

Insufficient

$30 \leq \mathrm{ng} / \mathrm{mL}$

Adequate

$100 \leq \mathrm{ng} / \mathrm{mL}$

Potential toxicity

Table 2. Demographic and clinical characteristics of patients

\begin{tabular}{|c|c|c|c|}
\hline & Patients with no DR & Patients with DR & P-value \\
\hline Gender (female/male), n & $16 / 14$ & $15 / 15$ & 0.73 \\
\hline Age (years), mean \pm SD & $58.30 \pm 7.23$ & $58.57 \pm 5.78$ & 0.75 \\
\hline FBS $(\mathrm{mg} / \mathrm{dL})$, mean $\pm \mathrm{SD}$ & $146.43 \pm 58.91$ & $164.37 \pm 88.98$ & 0.54 \\
\hline HbA1c (\%), mean \pm SD & $7.18 \pm 1.58$ & $7.90 \pm 2.25$ & 0.45 \\
\hline $\mathrm{Hb}(\mathrm{g} / \mathrm{dL})$, mean $\pm \mathrm{SD}$ & $14.20 \pm 1.22$ & $13.48 \pm 2.04$ & 0.78 \\
\hline $\mathrm{BUN}(\mathrm{mg} / \mathrm{dL})$, mean $\pm \mathrm{SD}$ & $13.66 \pm 3.98$ & $17.03 \pm 7.40$ & 0.41 \\
\hline $\mathrm{Cr}(\mathrm{mg} / \mathrm{dL})$, mean $\pm \mathrm{SD}$ & $1.02 \pm 0.25$ & $1.21 \pm 0.72$ & 0.33 \\
\hline Calcium (mg/dL), mean \pm SD & $9.26 \pm 0.45$ & $8.92 \pm 0.48$ & 0.511 \\
\hline Phosphorus (mg/dL), mean \pm SD & $3.75 \pm 0.42$ & $3.66 \pm 0.50$ & 0.416 \\
\hline $25-\mathrm{OH} D(\mathrm{ng} / \mathrm{mL})$ mean $\pm \mathrm{SD}$ & $15.61 \pm 9.40$ & $12.10 \pm 14.62$ & 0.012 \\
\hline
\end{tabular}

BUN, blood urea nitrogen; Cr, creatinine; DR, diabetic retinopathy; FBS, fasting blood sugar; HbA1c, hemoglobin A1c; Hb, hemoglobin; SD, standard deviation.

eight studies involving 13,435 participants showed vitamin D deficiency (serum $25(\mathrm{OH})$ D levels $<20$ $\mathrm{ng} / \mathrm{mL}$ ) increased the risk of $\mathrm{DR}(\mathrm{OR}=2.03,95 \% \mathrm{Cl}$ : $1.07-3.86)^{[20]}$ On the contrary, some studies found no differences in serum vitamin $D$ levels between diabetic patients with and without retinopathy. ${ }^{[16,18]}$

Evidence shows that vitamin D may affect the pathogenesis of DR via its effects on angiogenesis by changing the presence of hypoxiainducible products, such as vascular endothelial growth factor (VEGF). ${ }^{[9]}$ Ben-Shoshan et al found that 1, $25(\mathrm{OH})_{2} \mathrm{D}_{3}$ decreases the protein expression of both regulated hypoxia-inducible factor (HIF)-1 $1 \alpha$ subunit and the VEGF in human cancer cells. ${ }^{[21]}$ Vitamin $D$ reduces inflammatory products by decreasing the lymphocyte proliferation and cytokine production. ${ }^{[22]}$ Moreover, vitamin D deficiency influences the activity of tissue matrix metalloproteinase (MMPs) and C-reactive protein (CRP) that are involved in microangiopathies. ${ }^{[23]}$

We demonstrated that DR occurred more frequently in patients who were on insulin therapy than in those who used oral hypoglycemic agents to control their blood sugar $(P=0.005)$. This could be attributed to low vitamin $D$ concentration in these patients that could disturb the regulation of internal insulin secretion and increase the need for external insulin to control the blood sugar. ${ }^{[7,8]}$ In the present study, there were no statistically significant differences between groups regarding their $\mathrm{HbA}_{1} \mathrm{c}$, FBS, and the duration of diabetes. Furthermore, patients with DR had higher proteinuria than those without, which could demonstrate a correlation between DR and nephropathy.

Multiple studies reported that the presence of DR in type 2 diabetic patients was associated with longer duration of diabetes and higher levels of $\mathrm{HbA}_{1} \mathrm{c}^{[24-26]}$ However, in the present study, such correlation was not found; this disagreement could be explained by the small sample size of the current study that was not sufficient to detect these associations.

The cross-sectional design and the small sample size are the limitations of our study. Furthermore, 
outdoor activity time, patients' daily diet, and the mean blood pressure of patients, which are known to affect vitamin D levels, ${ }^{[27]}$ were not considered in the study.

Although there are several published studies on this topic with different results, to the best of our knowledge, no study on this subject is available from Fars Province, Iran. To improve our understanding of vitamin D deficiency and DR, a larger population-based study is required.

In conclusion, the current study showed that patients with type 2 diabetes and DR had lower serum vitamin $D$ levels than those without DR.

\section{Financial Support and Sponsorship}

Shiraz University of Medical Sciences.

\section{Conflicts of Interest}

There are no conflicts of interest.

\section{Acknowledgement}

The authors are grateful to Dr. Kazem Kamran, retinal specialist, and Dr. Roustsa Statistician, and all staff members of the Poostchi Ophthalmology Research Center involved in the study that contributed to the recruitment of participants and supported our activities.

\section{REFERENCES}

1. Geiss LS, Wang J, Cheng YJ, Thompson TJ, Barker L, Li $\mathrm{Y}$, et al. Prevalence and incidence trends for diagnosed diabetes among adults aged 20 to 79 years, United States, 1980-2012. JAMA 2014;312:1218-1226.

2. NCD Risk Factor Collaboration (NCD-RisC). Worldwide trends in diabetes since 1980: a pooled analysis of 751 population-based studies with 4.4 million participants. Lancet 2016;387:1513-1530.

3. Wan TT, Li XF, Sun YM, Li YB, Su Y. Recent advances in understanding the biochemical and molecular mechanism of diabetic retinopathy. Biomed Pharmacother 2015;74:145-147.

4. Bayani MA, Akbari R, Banasaz B, Saeedi F. Status of vitamin-D in diabetic patients. Caspian J Intern Med 2014;5:40-42.

5. Cangoz S, Chang YY, Chempakaseril SJ, Guduru RC, Huynh LM, John JS, et al. Vitamin D and type 2 diabetes mellitus. J Clin Pharm Ther 2013;38:81-84.

6. Mathieu C, Gysemans C, Giulietti A, Bouillon R. Vitamin D and diabetes. Diabetologia 2005;48:1247-1257.
7. Wimalawansa SJ. Associations of vitamin D with insulin resistance, obesity, type 2 diabetes, and metabolic syndrome. J Steroid Biochem Mol Biol 2018;175:177-189.

8. Michaud SE, Renier G. Direct regulatory effect of fatty acids on macrophage lipoprotein lipase: potential role of PPARs. Diabetes 2001;50:660-666.

9. Albert DM, Scheef EA, Wang S, Mehraein F, Darjatmoko $\mathrm{SR}$, Sorenson CM, et al. Calcitriol is a potent inhibitor of retinal neovascularization. Invest Ophthalmol Vis Sci 2007;48:2327-2334.

10. Zhang J, Upala S, Sanguankeo A. Relationship between vitamin $D$ deficiency and diabetic retinopathy: a metaanalysis. Can J Ophthalmol 2017;52:219-224.

11. Langlois K, Greene-Finestone L, Little J, Hidiroglou N, Whiting S. Vitamin D status of Canadians as measured in the 2007 to 2009 Canadian Health Measures Survey. Health Rep 2010;21:47-55.

12. Forrest KY, Stuhldreher WL. Prevalence and correlates of vitamin D deficiency in US adults. Nutr Res 2011;31:48-54.

13. Larijani B, Hossein-Nezhad A, Feizabad E, Maghbooli Z, Adibi $H$, Ramezani $M$, et al. Vitamin $D$ deficiency, bone turnover markers and causative factors among adolescents: a cross-sectional study. J Diabetes Metab Disord 2016;15:46.

14. Holick MF. Vitamin D deficiency. $N$ Engl J Med 2007;357:266-281.

15. Rhee SY, Hwang YC, Chung HY, Woo JT. Vitamin D and diabetes in Koreans: analyses based on the Fourth Korea National Health and Nutrition Examination Survey (KNHANES), 2008-2009. Diabet Med 2012;29:10031010.

16. Bonakdaran S, Shoeibi N. Is there any correlation between vitamin $\mathrm{D}$ insufficiency and diabetic retinopathy? Int $J$ Ophthalmol 2015;8:326-331.

17. Isaia G, Giorgino R, Adami S. High prevalence of hypovitaminosis $D$ in female type 2 diabetic population. Diabetes Care 2001;24:1496.

18. Alam U, Amjad Y, Chan AW, Asghar O, Petropoulos IN, Malik RA. Vitamin D deficiency is not associated with diabetic retinopathy or maculopathy. J Diabetes Res 2016;2016:6156217.

19. Payne JF, Ray R, Watson DG, Delille C, Rimler E, Cleveland $J$, et al. Vitamin D insufficiency in diabetic retinopathy. Endocr Pract 2012;18:185-193.

20. Luo BA, Gao F, Qin LL. The association between vitamin $D$ deficiency and diabetic retinopathy in type 2 diabetes: a meta-analysis of observational studies. Nutrients 2017;9:3.

21. Ben-Shoshan M, Amir S, Dang DT, Dang LH, Weisman Y, Mabjeesh NJ. 1alpha,25-dihydroxyvitamin D3 (Calcitriol) inhibits hypoxia-inducible factor-1/vascular endothelial growth factor pathway in human cancer cells. Mol Cancer Ther 2007;6:1433-1439.

22. Wu-Wong JR, Nakane M, Ma J, Ruan X, Kroeger PE. Effects of vitamin $D$ analogs on gene expression profiling in human coronary artery smooth muscle cells. Atherosclerosis 2006;186:20-28.

23. Timms PM, Mannan N, Hitman GA, Noonan K, Mills PG, Syndercombe-Court D, et al. Circulating MMP9, vitamin D and variation in the TIMP-1 response with VDR genotype: mechanisms for inflammatory damage in chronic disorders? QJM 2002;95:787-796.

24. Pan CW, Wang S, Qian DJ, Xu C, Song E. Prevalence, 
awareness, and risk factors of diabetic retinopathy among adults with known type 2 diabetes mellitus in an urban community in China. Ophthalmic Epidemiol 2017;24:188194.

25. Bansal P, Gupta R, Kotecha M. Frequency of diabetic retinopathy in patients with diabetes mellitus and its correlation with duration of diabetes mellitus. Med J Dr DY Patil Univ 2013;6:366-369.
26. Jenchitr W, Samaiporn S, Lertmeemongkolchai P, Chongwiriyanurak T, Anujaree P, Chayaboon D, et al. Prevalence of diabetic retinopathy in relation to duration of diabetes mellitus in community hospitals of Lampang. J Med Assoc Thai 2004;87:1321-1326.

27. Vashi PG, Lammersfeld CA, Braun DP, Gupta D. Serum 25hydroxyvitamin $D$ is inversely associated with body mass index in cancer. Nutr J 2011;10:51. 